This item was submitted to Loughborough's Research Repository by the author.

Items in Figshare are protected by copyright, with all rights reserved, unless otherwise indicated.

\title{
Opportunistic fountain coding with coordinative routing
}

\author{
PLEASE CITE THE PUBLISHED VERSION
}

https://doi.org/10.1109/LWC.2022.3147601

PUBLISHER

Institute of Electrical and Electronics Engineers

VERSION

AM (Accepted Manuscript)

\section{PUBLISHER STATEMENT}

(c) 2022 IEEE. Personal use of this material is permitted. Permission from IEEE must be obtained for all other uses, in any current or future media, including reprinting/republishing this material for advertising or promotional purposes, creating new collective works, for resale or redistribution to servers or lists, or reuse of any copyrighted component of this work in other works.

\section{LICENCE}

All Rights Reserved

\section{REPOSITORY RECORD}

Peng, Tong, Sangarapillai Lambotharan, Gan Zheng, and Mohammad Shikh-Bahaei. 2022. "Opportunistic Fountain Coding with Coordinative Routing". Loughborough University.

https://hdl.handle.net/2134/19213479.v1. 


\title{
Opportunistic Fountain Coding with Coordinative Routing
}

\author{
Tong Peng, Sangarapillai Lambotharan, Gan Zheng and Mohammad Shikh-Bahaei
}

\begin{abstract}
Fountain codes are widely employed to improve reliability and efficiency in wireless communication systems due to their simple coding mechanisms. In this letter, we propose an opportunistic decoding and recoding mechanism with coordinative routing algorithm for fountain codes to improve the expected transmission counts (ETX) performance and to reduce computational complexity of fountain decoding at each relay in multi-hop wireless sensor networks. The full recovery in existing cooperative fountain coded networks is replaced by a new partial decoding and recoding mechanism in which both the recovered and the unrecovered packets at the intermediate nodes are considered as recoding candidates. Numerical results show that the proposed algorithm is superior to the existing opportunistic routing-based fountain code schemes.
\end{abstract}

Index Terms-Partial fountain decoding, opportunistic routing, coordinative forwarding.

\section{INTRODUCTION}

In order to achieve high transmission reliability with low latency in wireless communications, linear network coding (LNC) is considered as a promising technique in practical networks [1]-[3]. In conventional LNCs, the original packets are linearly combined by using different encoding matrices before transmission and these matrices are sent with the coded packets for decoding at the destination. In order to achieve higher throughput and bandwidth efficiency, fountain codes $[4,5]$ with simple exclusive-OR (XOR) encoding mechanism are utilized as rateless network coding schemes. Thus, a transmitter can continuously send the coded packets without expecting an acknowledgement and all the original packets can be recovered at a receiver when adequate number of coded packets are accumulated.

Although fountain codes improve reliability of a network, certain performance metrics such as transmission latency and energy cost in modern multi-hop networks are not as good as that in a single-hop network [6]. Various opportunistic network coding strategies are proposed to resolve the above problems. In [7], a relay selection protocol that optimizes physical-layer opportunistic relaying and application-layer random LNC is proposed to reduce the packet wiretapping probability in twohop networks. The authors in [8] present a coding-aware routing strategy, where a sliding window-based inter-flow network coding scheme is combined with a routing protocol that maximizes the packet delivery probability, to improve coding efficiency and transmission latency performances of sparse

T. Peng, G. Zheng and S. Lambotharan are with Wolfson School of Mechanical, Electrical and Manufacturing Engineering, Loughborough University. T. Peng is also with College of Information Engineering, Zhejiang Ocean University, China. M. Shikh-Bahaei is with Centre for Telecommunications Research, Department of Engineering, King's College London, UK (emails: t.peng@lboro.ac.uk, g.zheng@lboro.ac.uk, s.lambotharan@lboro.ac.uk and m.sbahaei@kcl.ac.uk)

This work was supported by Engineering and Physical Sciences Research Council (EPSRC) under Grants EP/P022723/1 and EP/R006385/1, and Basic Scientific Research Fund of Provincial Universities under Grant No. 2021J016.

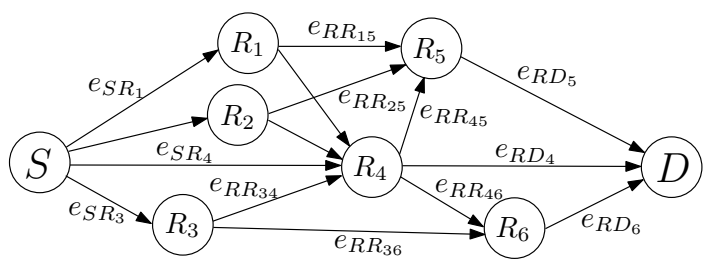

Fig. 1. An example of a multi-hop wireless sensor network with coordinative routing.

underwater wireless sensor networks. A multi-hop wireless network containing one single source and multiple destinations is considered in [9], and the authors employ the destination nodes in adaptive intermediate nodes selection for forwarding fountain coded packets to exploit different forwarding possibilities in the network. In [10], the authors study the nature of network coding-based opportunistic routing protocols in conventional networks and extend it to cognitive radio ad hoc networks to maximize the packet delivery ratio, where the whole network is divided into several small sub-networks with two hops. In all of the above research, either a forwarding mechanism employing multiple intermediate nodes or only one intermediate node is developed, the full-recovery of the original packets at least at one intermediate node is required. This may compromise the advantages of coordinative routing for end-to-end transmissions, such as the lower transmission latency and higher bandwidth efficiency.

In this letter, we propose an opportunistic decoding and recoding with coordinative routing (ODRCR) algorithm for fountain codes to improve transmission latency performance with reduced computational cost in multi-hop networks. The main difference between this work and the existing designs of opportunistic routing-based fountain codes (OR-FC) schemes [7]-[9] is that each relay node is not required to recover all original packets. A virtual node with full recovery capability will be selected for packets forwarding in the proposed algorithm so that full packet recovery is not required at any individual nodes. The unrecovered packets at an individual relay are used for fountain recoding with the recovered ones. In order to reduce the end-to-end latency and computational complexity, both the expected transmission count (ETX) and decoding complexity are employed as optimization metrics in the proposed ODRCR algorithm. To the best of the authors' knowledge, this is the first work to discuss the implementation of fountain recoding with partially decoded packets and to adopt this in opportunistic routing algorithms.

\section{System Model}

We consider a multi-hop wireless sensor network (WSN) where a source node $S$ delivers $N$ packets $\mathbf{p}=\left[p_{1}, \cdots, p_{N}\right]$ to a destination node $D$ via multiple hops among $M$ relay 
nodes $\left\{R_{1}, R_{2}, \cdots, R_{M}\right\}$. Each individual link between a pair of nodes is modeled as a packet erasure channel and presented by an independent packet erasure coefficient $e_{A B_{i j}}$, where $A, B \in\{S, R, D\}, i, j \in\{1, \ldots, M\}$ and $i \neq j$. The forwarded packets are randomly erased during the transmissions according to the value of $e_{A B_{i j}}$ which is constant during a transmission interval. Note that the index $j$ is removed when $A, B=S, R$. A system example is illustrated in Fig. 1 .

The original packets $\mathbf{p}$ are coded by a fountain encoding matrix in Raptor codes [5] before the transmission, as described by

$$
p^{F C}=\mathbf{g}^{F C} \mathbf{p}=\mathbf{g}^{L T} \mathbf{p}^{I T}=\mathbf{g}^{L T} \mathbf{G}^{I T} \mathbf{p},
$$

where $p^{F C}$ denotes a fountain coded packet. $\mathbf{p}^{I T}=\left[\mathbf{p} \mid \mathbf{p}^{\text {Pre }}\right]$ stands for an intermediate symbol vector with a length of $N_{I T}=N+N_{\text {Pre }}$, containing the original packet vector $\mathbf{p}$ and the precoded packet vector $\mathbf{p}^{\text {Pre }}$. Note that the value of $N_{I T}$ for the same $N$ varies according to different coding schemes. $\mathrm{g}^{L T}$ denotes a $1 \times N_{I T}$ binary outer LT encoding vector, and $\mathbf{G}^{I T}=\left[\mathbf{I} \mid \mathbf{G}^{P r e}\right]^{T}$ stands for an $N_{I T} \times N$ binary intermediate precoding matrix in Raptor codes. [. $]^{T}$ stands for the transpose of a matrix. The performance of different fountain codes varies according to their distribution and feedback strategies, and we focus on how the proposed opportunistic decoding and coordinative relaying are implemented instead of discussing optimization of their distribution.

Independent decoding starts when $K$ packets are received at each relay node by different decoding algorithms, such as maximum likelihood (ML), Gaussian elimination [5] and unipartite graph algorithms [11]. In order to unify the expression of encoding and decoding, we use a decoding matrix here to represent the decoding procedure in the above algorithms, given by

$$
\hat{\mathbf{p}}=\mathbf{G}^{D e c} \mathbf{p}^{F C}=\mathbf{G}^{D e c} \mathbf{G}^{L T} \mathbf{G}^{I T} \mathbf{p}
$$

where $\mathbf{G}^{D e c}$ stands for the $\hat{N} \times K$ decoding matrix, and $\hat{N}$ denotes the number of recovered packets. $\mathbf{G}^{L T}=$ $\left[\mathbf{g}_{1}^{L T}, \cdots, \mathbf{g}_{K}^{L T}\right]^{T}$ stands for the binary $K \times N_{I T}$ LT outer coding matrix. The transmission stops when a receiver node recovers all the original packets, followed by recoding at the relay nodes using (1). Therefore, the packets forwardingdecoding-recoding loop is implemented in each hop until all the original packets are recovered at the destination node $D$. The decoding complexity at each node is proportional to the dimension of $\mathbf{p}^{F C}$ and can be calculated by $O(N+\epsilon)$, where $\epsilon$ stands for the overhead in conventional FC schemes.

\section{The Proposed Opportunistic Fountain Coding With CoOrdinative Routing Algorithm}

In existing opportunistic decoding strategies among multiple forwarders [9, 12], the recoding and forwarding are ended when at least one receiver node can recover all the original packets. Such requirement is adopted to eliminate the decoding failure due to the lack of necessary packets, but the transmission latency may increase due to the ignorance of recovery status at the other intermediate nodes [13]. In the proposed opportunistic decoding and recoding with coordinative

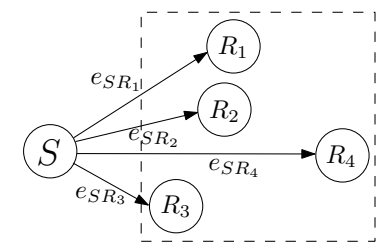

(a)

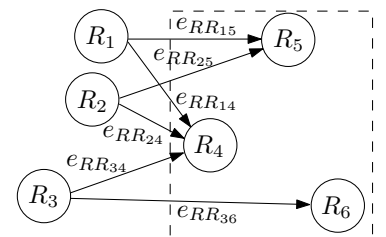

(b)
Fig. 2. An example of virtual node (VN) set in different single hop scenarios: (a) $S$ to the $V N$ set of $\left\{R_{1}, R_{2}, R_{3}, R_{4}\right\}$; (b) the $V N$ set of $\left\{R_{1}, R_{2}, R_{3}\right\}$ to the $V N$ set of $\left\{R_{4}, R_{5}, R_{6}\right\}$.

routing (ODRCR) algorithm, the relay nodes which can hear from a transmitter form a virtual relaying set with multiple distributed and independent antennas. This set is defined as the virtual relaying node set (VRNS) in this letter. A VRNS contains several virtual nodes (VNs) which are established by different combinations of the relays within this VRNS. When a VN contains all required coded packets for original packet recovery, the transmission stops and each relay node in the VN implements independent decoding. A benefit achieved by the above VRNS structure is to improve the transmission latency by eliminating the waiting time of complete recovery at any individual relay nodes. Existing feedback strategies in feedback-based fountain codes [14] can be used for reception status sharing among VNs instead of introducing additional information exchanges. Each bit in the feedback notice is binary and it indicates whether a coded packet is successfully received or not. An example is given as follows.

Example 1. A snapshot of the multi-hop transmissions from $S$ is illustrated in Fig. 2. In the first hop illustrated in Fig. 2(a), $S$ broadcasts to all relays and these four relays form a reception VRNS. The reception status at each relay are shared among the relays and $S$ by using a periodical feedback mechanism. Since $S$ knows the complete encoding matrix, an end-of-transmission notice is sent to the reception VRNS when enough coded packets are accumulated for all the original packet recovery. Independent decoding is implemented at each relay node and one VN is selected among all $\sum_{i=1}^{4}\left(\begin{array}{l}4 \\ i\end{array}\right)=15$ possible combinations of VNs in the VRNS containing $R_{1}, R_{2}$, $R_{3}$ and $R_{4}$ for packets forwarding as illustrated in Fig. 2(b). Note that $R_{4}$ can stay in the reception VNRS in the next hop if it is not selected for packets forwarding.

We now present how to select the optimal VNs by using the proposed ODRCR algorithm. Since independent decoding is implemented at each relay node, the decoded packet vector at the $i^{t h}$ relay in the $j^{t h} \mathrm{VN}$ can be derived as

$$
\hat{\mathbf{p}}_{R V_{i j}}=\mathbf{G}_{R V_{i j}}^{D e c} \mathbf{p}_{R V_{i j}}^{F C}=\left[\mathbf{G}_{R V_{i j}}\right]^{T} \mathbf{p}=\left[\hat{\mathbf{I}}_{R V_{i j}} \mid \mathbf{G}_{R V_{i j}}^{O D}\right]^{T} \mathbf{p},
$$

where $\mathbf{G}_{R V_{i j}}^{D e c}$ denotes a partial decoding matrix and it can be rewritten in a form of binary generation matrix $\mathbf{G}_{R V_{i j}}$ with a dimension of $N \times N_{R V_{i j}}$, and $N_{R V_{i j}}$ denotes the number of independent columns which includes $\hat{N}_{R V_{i j}}$ columns with only a 1 in each column and $N_{R V_{i j}}^{O D}=N_{R V_{i j}}-\hat{N}_{R V_{i j}}$ columns with multiple 1s. $\hat{\mathbf{I}}_{R V_{i j}}$ stands for an $N \times \hat{N}_{R V_{i j}}$ 
pseudo identity binary matrix with a 1 in each column, and $\mathbf{G}_{R V_{i j}}^{O D}$ is an $N \times N_{R V_{i j}}^{O D}$ binary matrix which represents the XOR of unrecovered packets at the $i^{\text {th }}$ relay. Note that $\hat{N}_{R V_{i j}} \in[0, N]$, where $\hat{N}_{R V_{i j}}=0$ indicates not enough coded packets are accumulated for packet recovery at this relay and $\hat{N}_{R V_{i j}}=N$ indicates all the original packets are recovered, then $\hat{\mathbf{I}}_{R V_{i j}}=\mathbf{I}_{N \times N}$ is an identity matrix and $\mathbf{G}_{R V_{i j}}^{O D}=\mathbf{0}$. The decoding complexity of the VN selection criterion in (3) is proportional to the number of partial decoded packets and can be calculated with complexity $O\left(\hat{N}_{R V_{i j}}+\epsilon_{R V_{i j}}\right)$, where $\epsilon_{R V_{i j}}$ stands for the overhead. According to [5], the partial decoding complexity is less than or equal to that of the full decoding complexity in conventional FC schemes due to $\hat{N}_{R V_{i j}} \leq N$ and $\epsilon_{R V_{i j}} \leq \epsilon$. According to (3), the accumulated packets at a VN with $L$ relays can be written as

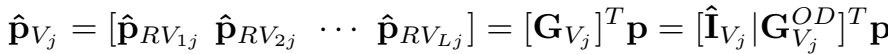

$$
\begin{aligned}
& =\left[\begin{array}{llll}
{\left[\hat{\mathbf{I}}_{R V_{1 j}}\right.} & \cdots & \hat{\mathbf{I}}_{R V_{L j}}
\end{array}\right] \mid\left[\begin{array}{lll}
\mathbf{G}_{R V_{1 j}}^{O D} & \cdots & \mathbf{G}_{R V_{L j}}^{O D}
\end{array}\right]^{T} \mathbf{p} .
\end{aligned}
$$

It is clear that $\mathbf{G}_{V_{j}}$ is a binary partial decoding matrix with redundancy information due to the repetitive accumulated packets at different relay nodes. In order to eliminate the redundancy of the generation matrix and reduce the probability of transmission obstruction in the network, we propose a new opportunistic decoding and recoding strategy for establishing a VN for coordinate packets forwarding. We firstly define a VRNS containing all VN combinations, given by

$$
\begin{aligned}
\{V R N S\} & =\left\{V N_{1}, V N_{2}, \cdots, V N_{J}\right\} \\
& =\left\{\left\{R_{1}\right\}, \cdots,\left\{R_{L}\right\},\left\{R_{1}, R_{2}\right\},\left\{R_{1}, R_{3}\right\}, \cdots\right\},
\end{aligned}
$$

where $J=\sum_{i=1}^{L}\left(\begin{array}{c}L \\ i\end{array}\right)$ denotes all possible combinations of relay nodes within a VRNS. After the independent decoding at each relay of the $\mathrm{VN}$, the recovered and unrecovered packets in this $\mathrm{VN}$ will be treated as recoding candidates and the unrecovered packets are directly treated as intermediate coded packets. The same encoding degree distribution can be used to keep the average degree of the recoded packets low in order to limit the decoding complexity, and the recoded packets can be constructed as

$$
\begin{gathered}
\mathbf{p}_{V_{j}}^{R e F C}=\mathbf{G}_{V_{j}}^{F C} \hat{\mathbf{p}}_{V_{j}}=\mathbf{G}_{V_{j}}^{L T} \mathbf{G}_{V_{j}}^{I T}\left[\mathbf{G}_{V_{j}}\right]^{T} \mathbf{p}=\mathbf{G}_{V_{j}}^{R e F C} \mathbf{p}, \\
\text { for } j=1, \cdots, J,
\end{gathered}
$$

where $[\cdot]^{\text {ReFC }}$ stands for recoding and $[\cdot]_{V_{j}^{q}}$ stands for the $j^{\text {th }}$ VN of a VRNS. Note that the recoding is independent at each relay so that we have $\mathbf{G}_{V_{j}}^{L T}=\left[\mathbf{G}_{R V_{1 j}}^{L T} \cdots \mathbf{G}_{R V_{L j}}^{L T}\right]^{T}$ and $\mathbf{G}_{V_{j}}^{I T}=\left[\mathbf{G}_{R V_{1 j}}^{I T} \cdots \mathbf{G}_{R V_{L j}}^{I T}\right]^{T}$, where $\mathbf{G}_{R V_{l j}}^{L T}$ and $\mathbf{G}_{R V_{l j}}^{I T}$ denote the LT and intermediate encoding matrix at the $l^{\text {th }}$ relay node of the $j^{t h} \mathrm{VN}$, respectively, and $L$ is the total number of relays within this VN. According to the definition of $\mathrm{VN}$, the recoding matrix at each relay $\mathbf{G}_{R V_{l j}}^{L T} \mathbf{G}_{R V_{l j}}^{I T}$ does not necessarily contain the full original packet recovery capability, but the joint recoding matrix $\mathbf{G}_{V_{j}}^{R e F C}$ should. In order to guarantee the full original packet recovery at a $\mathrm{VN}$, we have the following lemma

Lemma 1. Given a recoding matrix $\mathbf{G}_{V}^{R e F C}$ for a $V N$, all $N$ packets can be recovered if and only if $\mathbf{G}_{V}^{R e F C}$ is a full-rank matrix with the rank of $N$.
Proof. The recoding matrix $\mathbf{G}_{V}^{R e F C}$ contains two different elements. The first one is recoding matrix $\mathbf{G}_{V}^{F C}$, which is a $K \times N_{V}$ encoding matrix calculated by the product of the $K \times N_{I T}$ outer LT encoding matrix $\mathbf{G}_{V}^{L T}$ and the $N_{I T} \times N_{V}$ inner intermediate encoding matrix $\mathbf{G}_{V}^{I T}$, where $N_{V}=$ $\sum_{l=1}^{L} N_{R V_{l}}$ denotes the number of accumulated packets at the relays in this $\mathrm{VN}$. According to [5], the minimum number of independent linear equations to recover the accumulated packets is $N_{V}$.

The second element is the opportunistic decoding matrix, which can be written as a generation matrix $\mathbf{G}_{V}$. According to (4), the dimension of $\mathbf{G}_{V}$ for a $\mathrm{VN}$ is $N \times N_{V}$. Therefore, in order to recover $N$ packets, such generation matrix contains at least $N$ independent linear equations. In this case, this $K \times N$ recoding matrix $\mathbf{G}_{V}^{R e F C}$ should be a full-rank matrix with the rank of $N$ for full-recovery of all the original packets.

Since the above Lemma can be satisfied easily by the VNs containing a high number of relays, we now focus on how to select the optimal VNs containing the full original packet recovery capability with the lowest decoding complexity. The decoding of the recoded packets $\mathbf{p}_{R V_{i j}}^{R e F C}$ is given in (2) and in order to minimize the calculation complexity of the decoding, the dimension of the product of decoding and recoding matrices should be minimized, as described below

$$
\begin{gathered}
\min _{j} \mathcal{D}\left\{\mathbf{G}_{V_{j}}^{D e c} \mathbf{G}_{V_{j}}^{R e F C}\right\}, \\
\text { s.t. } \mathcal{R}\left\{\mathbf{G}_{V_{j}}^{R e F C}\right\} \geq N, \forall V N_{j} \in\{V R N S\},
\end{gathered}
$$

where $\mathcal{D}\{\cdot\}$ and $\mathcal{R}\{\cdot\}$ stand for the dimension and rank of a matrix, respectively. According to (1) and (5), the binary recoding matrix $\mathbf{G}_{V_{j}}^{R e F C}$ in a $\mathrm{VN}$ represents the way to apply XOR among the packet candidates and it can be written as $\mathbf{G}_{V_{j}}^{R e F C}=\left[\hat{\mathbf{I}}_{V_{j}} \mid \mathbf{G}_{V_{j}}^{O D}\right]^{T}$, where $\hat{\mathbf{I}}_{V_{j}}$ and $\mathbf{G}_{V_{j}}^{O D}$ stand for the recoded matrix with degree of 1 and that greater than 1 , respectively. Note that repetitive columns may exist in $\mathbf{G}_{V_{j}}^{R e F C}$, especially in $\hat{\mathbf{I}}_{V_{j}}$ due to the decoding capability of the same packets at different relays. These repetitive packets should not be removed in recoding cause they are accumulated at different relays. In this case, the number of recoded packets in a VN should be minimized by selecting the VNs with small recoding matrix dimension and full rank, which leads to the optimization in (6).

Having presented the first criterion of the proposed ODRCR algorithm above, we now illustrate how to improve the transmission latency in a multi-hop network by using a coordinative routing strategy. Since the reception status at each relay is shared within a $\mathrm{VN}$, then given a $\mathrm{VN}$ containing $L_{s}$ number of relays, the transmission latency can be expressed by the total expected transmission counts (ETX) of the recoded packets from this VN to its reception VRNS, mathematically given by

$$
E T X_{V_{j}}=\frac{\mathcal{L}\left\{\mathbf{p}_{V_{j}}^{R e F C}\right\}}{1-e_{V_{j}}}=\sum_{l_{s}=1}^{L_{s}} \frac{\mathcal{L}\left\{\mathbf{p}_{l_{s}}^{R e F C}\right\}}{1-\prod_{l_{r}=1}^{L_{r}} e_{R R_{l_{s} l_{r}}}},
$$

where $L_{r}$ stands for the number of relays in the reception VRNS, and $\mathcal{L}\{\cdot\}$ denotes the length of a vector. One problem of the above latency expression is the unfairness to the VNs containing higher number of relays, and it can be resolved 
by orthogonal frequency reutilization in which different frequencies are assigned to the relays to achieve simultaneous transmissions, like the implementation in [9]. Therefore, the expression of the transmission latency (TL) in (7) can be replaced by the maximum ETX between a pair of VNs, given by

$$
\begin{gathered}
T L_{V_{j k}}=\max _{k} E T X_{V_{j k}}=\max _{k} \frac{\mathcal{L}\left\{\mathbf{G}_{V_{j}}^{L T} \mathbf{G}_{V_{j}}^{I T}\left[\mathbf{G}_{V_{j}}\right]^{T} \mathbf{p}\right\}}{1-\prod_{l_{r}=1}^{L_{r}^{(k)}} e_{R R_{l_{s} l_{r}}}}, \\
\text { for } k=1,2, \cdots, K,
\end{gathered}
$$

where $K=\sum_{i=1}^{L_{r}}\left(\begin{array}{c}L_{r} \\ i\end{array}\right)$ stands for the number of VNs in the reception VRNS, and $L_{r}^{(k)}$ stands for the number of relays within the $k^{t h} \mathrm{VN}$. Then given a transmission $\mathrm{VN}$, the transmission latency can be reduced by the following reception VN selection algorithm

$$
\begin{gathered}
\min _{k} T L_{V_{j k}}, \\
\text { s.t. } \mathcal{R}\left\{\mathbf{G}_{V_{j k}}^{R e F C}\right\}=N, \forall V N_{k} \in\{V R N S\} .
\end{gathered}
$$

In order to select the optimal VNs for each hop according to the criteria in (6) and (9), a table containing all VRNSs in each hop is established according to their selection priorities. Initially, the value of priority for each $\mathrm{VN}$ is equal to 0 . A number of pilot symbols are then sent out in the network to obtain the values of $\mathcal{D}\left\{\mathbf{G}_{V_{j k}}^{D e c} \mathbf{G}_{V_{j k}}^{R e F C}\right\}$ and $T L_{V_{j k}}$ for each VN. Different selection priority values are assigned to the VNs in each hop according to (6) and (9) and those VNs containing the highest value are used for packets forwarding. According to (9), the computational complexity of the proposed ODRCR algorithm $O\left(\sum_{l=1}^{L_{r}}\left(\hat{N}_{R V_{i j}}^{(l)}+\epsilon_{R V_{i j}}^{(l)}\right)\right) \leq O(L(N+\epsilon))$, where the righthand side of the inequality is the computational complexity of conventional OR-FC schemes and $L$ denotes the number of relays employed for packets forwarding. The computational complexities are equal if and only if only one relay is selected, in which case both the proposed algorithm and the conventional OR-FC schemes will lose the advantage of coordinative relaying. We summarize the above ODRCR algorithm for the optimal VN selection in Algorithm 1.

\section{Simulation Results}

The simulation results of applying different network coded opportunistic routing algorithms in a multi-hop network are presented in this section. The network model considered in the simulation is a multi-hop network, where a source $S$ transmits 100 packets to a destination $D$ via multiple hops among VNs and each VN contains different number of relays. Raptor codes [5] are used at each transmitter and the size of the encoder is set to $g=10$. A conventional optimal routing selection scheme employed in [13] that employs a single relay at each hop as well as a cooperative multiple relaying (CMR) scheme [9] that employs all available relays at each hop are used as the benchmarks in the simulation. The total transmission power is fixed for each hop, and it is evenly assigned to the relays that are employed for packets forwarding in different schemes for fairness. As mentioned in Section I, we assume the wireless channels are block fading and the $\mathrm{VN}$ table update is implemented after each block fading period.
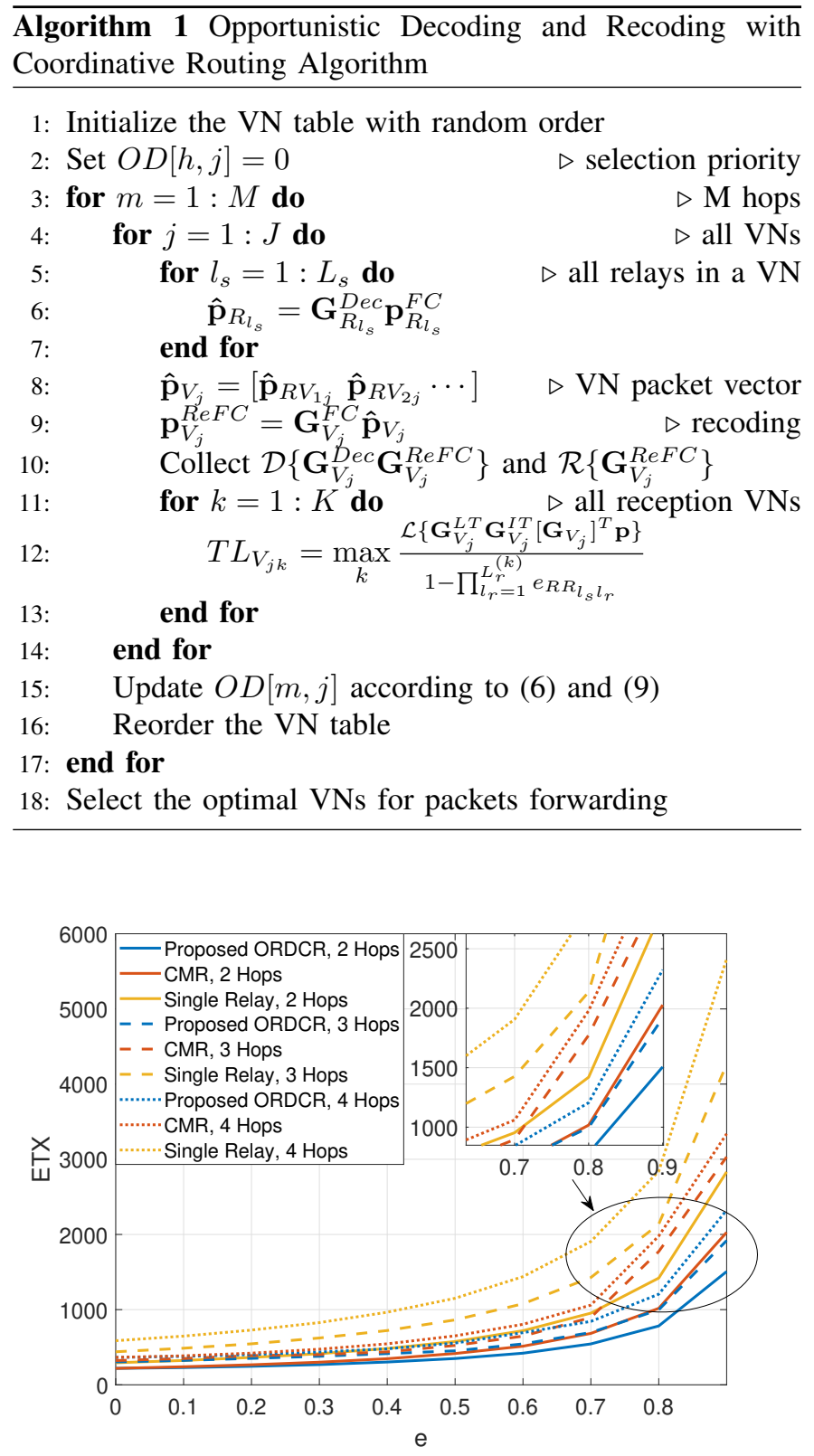

Fig. 3. Expected transmission count of different algorithms for various packet erasure probabilities, two relays per hop.

In Fig. 3 and 4, expected transmission count (ETX) and decoding complexity performances comparison for different number of hops are provided, respectively. The number of relays per hop is fixed to 2 and the packet loss probability $e$ in each link is also fixed during the transmission. As seen from Fig. 3, a higher ETX is required in all schemes with the increase of the packet loss probability. The number of hops has significant impact on ETX performance. With the help of coordinative relaying, CMR and the proposed ODRCR algorithms need much less ETX compared to the single relay scheme. As illustrated in the figure, the proposed ODRCR algorithm needs much less ETX to complete the transmission compared to the benchmarks, especially when the number of hops is high and the channel quality is poor. The decoding complexity is measured by the sum of the number of additions 

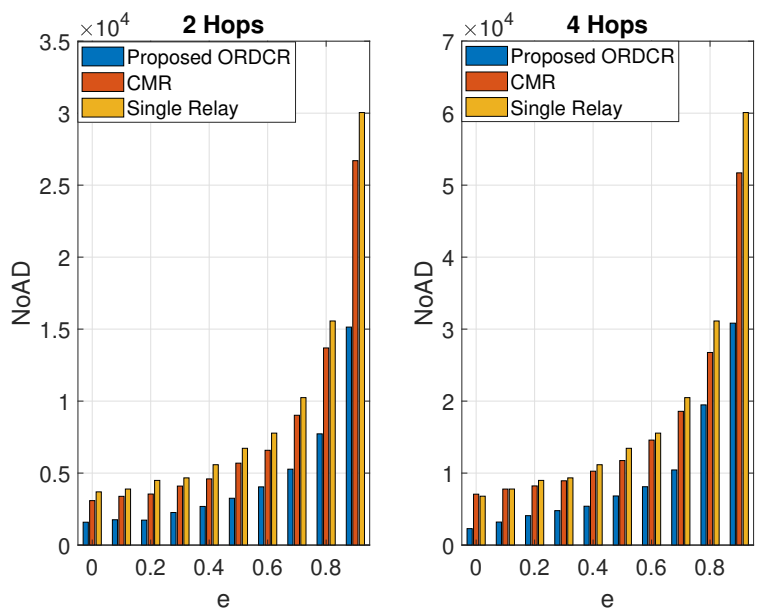

Fig. 4. Decoding complexity of different algorithms in different packet loss probabilities, two relays per hop.

(NoAD) of Gaussian elimination algorithm in the simulation. As seen in Fig. 4, NoAD in the single relay scheme is higher than the schemes employing coordinative relaying in different transmission circumstances. Compared to the CMR scheme where full packet recovery is required at one relay node, partial decoding in the proposed ODRCR algorithm contains much lower decoding complexity, which contributes to the latency improvement in multi-hop transmissions especially when communication channels undergo bad propagation conditions.
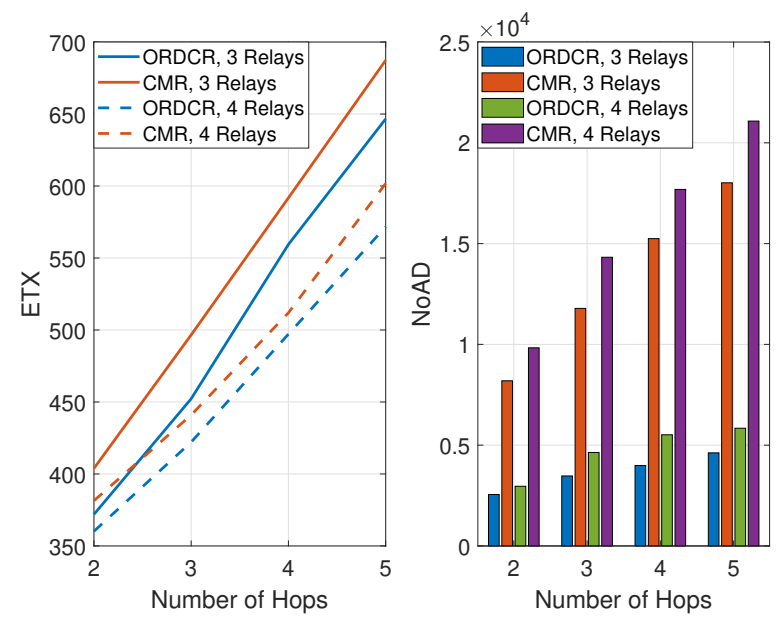

Fig. 5. Performance of the algorithms in multiple relays and multi-hop networks.

We then evaluate the transmission latency and decoding complexity performances of different coordinative relaying schemes with random selection of packet loss probabilities, and the results are illustrated in Fig. 5. In the simulation, the packet loss probability in each link is randomly generated between $50 \%$ and $80 \%$. We consider packets forwarding in a general multi-hop network with different number of relays in each hop. As illustrated in the figure, both ETX and NoAD performances get worse with the increase in the number of hops and the proposed ODRCR algorithm outperforms the
CMR scheme. With lower ETX, the decoding complexity of the proposed ODRCR algorithm is $1 / 4^{t h}$ of that of CMR scheme, which is achieved by partial decoding at each relay node in a VN. When more relays are employed in each hop, ETX performances in both CMR and the proposed ODRCR algorithms decrease due to the increased relaying opportunities and the gap between these schemes also shrinks.

\section{CONCLUSION}

In this work, we proposed an opportunistic recoding mechanism with partial decoding for fountain codes to reduce decoding complexity at each relay in multi-hop WSNs. Based on this scheme, we presented a coordinative relay selection algorithm to improve ETX performance. The simulation results indicate that the proposed algorithm outperforms the benchmark schemes and provides an efficient method to reduce the ETX and decoding complexity in multi-hop WSNs. However, with the increased number of relays in the network, the partial decoding complexity at each relay and the $\mathrm{VN}$ selection decision delay could hinder the performance of the proposed algorithm. New techniques and designs to resolve the above issues, particularly for adopting our algorithms to large scale networks, will be considered in our future studies.

\section{REFERENCES}

[1] S.-Y. R. Li, R. W. Yeung and N. Cai, "Linear Network Coding," IEEE Trans. Inform. Theory, vol. 49, no. 2, pp. 371-381, Feb. 2003.

[2] P. R. Mane, S. G. Adiga, and M. S. Kumar, "Performance evaluation of random linear network coding using a Vandermonde matrix," Phys. Commun., vol. 10, no. 1, pp. 24-30, Mar. 2014.

[3] W. L. CHen, F. Lu and Y. Dong, "Improved Expression for Rank Distribution of Sparse Random Linear Network Coding," IEEE Commun. Lett., Early Access, pp. 1472-1476, Dec. 2020.

[4] M. Luby, "LT Codes," in Proc. The 43rd Annual IEEE Symposium on Foundations of Computer Science, Vancouver, BC, Canada, Nov. 2002.

[5] A. Shokrollahi, "Raptor Codes," IEEE Trans. Inform. Theory, vol. 52, no. 6, pp. 2551-2567, Jun. 2006.

[6] X. Zhou, X. Yang, J. Ma, etc., "Energy Efficient Smart Routing Based on Link Correlation Mining for Wireless Edge Computing in IoT," IEEE Internet of Things Journal, Early Access, May 2021.

[7] A. S. Khan and I. Chatzigeorglou, "Opportunistic Relaying and Random Linear Network Coding for Secure and Reliable Communication," IEEE Trans. Wireless Commun., vol. 17, no. 1, pp. 223-234, Jan. 2018.

[8] D. Zhao, G. Lun and R. Xue, "Coding-Aware Opportunistic Routing for Sparse Underwater Wireless Sensor Networks," IEEE Access, vol. 9, pp. 50170-50187, Mar. 2021.

[9] S. S. Borkotoky and M. B. Pursley, "Fountain-Coded Broadcast Distribution in Multiple-Hop Packet Radio Networks," IEEE/ACM Trans. Netw., vol. 27, no. 1, pp. 29-41, Feb. 2019.

[10] X. Tang, J. Zhou, S. Xiong, etc., "Geographic Segmented Opportunistic Routing in Cognitive Radio Ad Hoc Networks Using Network Coding," IEEE Access, vol. 6, pp. 62766-62783, Oct. 2018.

[11] J. Huang, Z. Fei, C. Cao and M. Xiao, "Design and Analysis of Online Fountain Codes for Intermediate Performance," IEEE Trans. Commun., vol. 68 , no. 9 , pp. 5313-5325, Sept. 2020.

[12] J. Huang, Z. Fei, C. Cao, M. Xiao and X. Xie, "Reliable Broadcast Based on Online Fountain Codes," IEEE Commun. Lett., vol. 25, no. 2, pp. 369-373, Feb. 2021.

[13] J. Lou, X. Yuan, S. Kompella, etc., "Boosting or Hindering: AoI and Throughput Interrelation in Routing-Aware Multi-Hop Wireless Networks," IEEE/ACM Trans. Netw., vol. 29, no. 3, pp. 1008-1021, Feb. 2021.

[14] L. Sun and H. Xu, "Fountain-Coding-Based Secure Communications Exploiting Outage Prediction and Limited Feedback," IEEE Trans. Vehicu. Techno., vol. 68, no. 1, pp. 740-753, Jan. 2019. 